\title{
Implementation of high sensitivity troponin in a veterans health system: lessons learned
}

\author{
David E. Winchester ${ }^{1,2}$ \\ 'Malcom Randall VAMC Cardiology Section, Gainesville, FL 32608, USA. \\ 2University of Florida College of Medicine, Gainesville, FL 32610, USA.
}

Correspondence to: Prof./Dr. David E. Winchester, Malcom Randall VAMC Cardiology Section, Gainesville FL 32608, USA; University of Florida College of Medicine, Gainesville FL 32610, USA. E-mail: david.winchester@va.gov

How to cite this article: Winchester DE. Implementation of high sensitivity troponin in a veterans health system: lessons learned. Vessel Plus 2021;5:1. http://dx.doi.org/10.20517/2574-1209.2020.53

Received: 25 Sep 2020 First Decision: 3 Dec 2020 Revised: 4 Dec 2020 Accepted: 7 Dec 2020 Published: 8 Jan 2021

Academic Editor: Deborah B. Diercks Copy Editor: Miao Zhang Production Editor: Jing Yu

\begin{abstract}
Aim: To describe the implementation of a high sensitivity (hsTn) assay at a large academically affiliated Veteran Affairs medical center.

Methods: Approximately 6 months time was utilised for adoption of a new hsTn assay in clinical practice at our facility. We assembled a multidisciplinary team of front-line clinicians to determine which actions would best prepare the facility for the transition. Identified goals included: maximizing sensitivity of the assay, minimizing confusion for clinicians, making adoption as easy as possible for the end users, and encouraging clinicians to use uniform terminology while referring to troponin results. One month prior to the new assay initiation, educational interventions included live learning sessions, pre-recorded lectures, slide decks, and printed materials. Communication to the entire clinical staff was accomplished through staff meetings and email announcements. An abbreviated "top things to remember about hsTn list" was widely shared.
\end{abstract}

Results: After adoption, the cardiology service received minimal consultation requests from clinicians unaware of the change or uncomfortable with how to interpret the hsTn results. In quantitative assessment, we observed no change in the rate of cardiology consultations, electrocardiograms or cardiac stress tests.

Conclusion: A multidisciplinary implementation focused on application by the bedside and ample education were effective in creating a seamless transition to hsTn.

\footnotetext{
(@) ( $)$

(C) The Author(s) 2021. Open Access This article is licensed under a Creative Commons Attribution 4.0 International License (https://creativecommons.org/licenses/by/4.0/), which permits unrestricted use, sharing, adaptation, distribution and reproduction in any medium or format, for any purpose, even commercially, as long as you give appropriate credit to the original author(s) and the source, provide a link to the Creative Commons license, and indicate if changes were made.
}

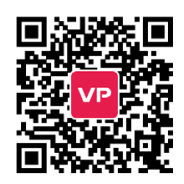


Keywords: Troponin, multidisciplinary communication, clinical laboratory techniques, acute coronary syndrome, cardiology

\section{INTRODUCTION}

Cardiac troponin is an invaluable serum assay used to detect myocardial damage and aids in diagnosing acute coronary syndromes (ACS). Troponin assays have steadily improved for decades and are crossing a threshold whereby cardiac troponin will be detectable in the majority of a normal population. These high sensitivity cardiac troponin (hsTn) assays have the potential to cause substantial confusion if adopted in a haphazard fashion.

While hsTn assays have been available in Europe for years, they have only recently received approval for use in the United States (US). Evidence suggests that the adoption of hsTn in Europe has taken several years, and is still not complete ${ }^{[1]}$. Current estimates of adoption are not available for US facilities, but it seems reasonable to expect a similar timeframe for adoption. Whether or not facilities are enthusiastic about adopting hsTn, manufacturers are unlikely to continue selling the older, less sensitive assays. Leadership at every facility that uses troponin should begin preparing for the transition to hsTn. Literature from Europe is informative for planning this transition; while recommendations for US have been published, primary literature on the US implementation remains limited ${ }^{[2]}$.

In this manuscript, we describe the extensive planning and implementation process used to adopt hsTn in a large, academically-affiliated Veterans Health System. Our timeline and lessons learnt will be shared to demonstrate how to use a multidisciplinary approach to identify resources, prioritize goals, create and distribute educational materials, and minimize disruptions to everyday clinical practice.

\section{METHODS}

\section{Background}

The North Florida/South Georgia Health System is a network of 2 medical centers and 11 outpatient clinics spanning 50 counties and 40,000 square miles, providing care for 130,000 Veterans with over 12,000 hospital admissions annually. Our health system elected to transition from the Elecsys Troponin T (Roche Diagnostics US, Indianapolis IN) to the Elecsys Troponin T-hs assay (Roche Diagnostics US, Indianapolis IN). Discussion of implementation will be focused on the primary medical center, Malcom Randall VAMC (MRVAMC), which is located in Gainesville, FL and accounts for the substantial majority of inpatient care in our health system. MRVAMC is academically affiliated with the University of Florida College of Medicine (UFCOM). The academic hospital affiliated with the UFCOM implemented a hsTn assay approximately one year prior to MRVAMC. Many, but not all, academic departments and graduate medical education programs share resources between the University and medical center; cardiology and internal medicine trainees rotate through MRVAMC, emergency medicine does not.

When a patient with suspected ACS is seen in our facility, the first point of medical contact is with the Emergency Department (ED). Initial evaluation includes history taking, physical examination, electrocardiogram, and measurement of cardiac biomarkers. If the patient is felt to be having or be at high risk for ACS after the initial evaluation, they will be admitted to the hospital medicine service. Admission is often under an observation status and the patient may be placed on a non-teaching teaching or teaching (with internal medicine residents) service. Cardiologists serve as consultants and do not have an independent admission service. Cardiology fellows are often the first contact with a patient if a consult is requested. A cardiology attending is directly available to anyone in the health system $24 \mathrm{~h}$ a day via an oncall cellular telephone. Our coronary catheterization laboratory is also available $24 \mathrm{~h}$ a day and is one of the highest volume centers for diagnostic and interventional cardiovascular procedures in the VA network. 


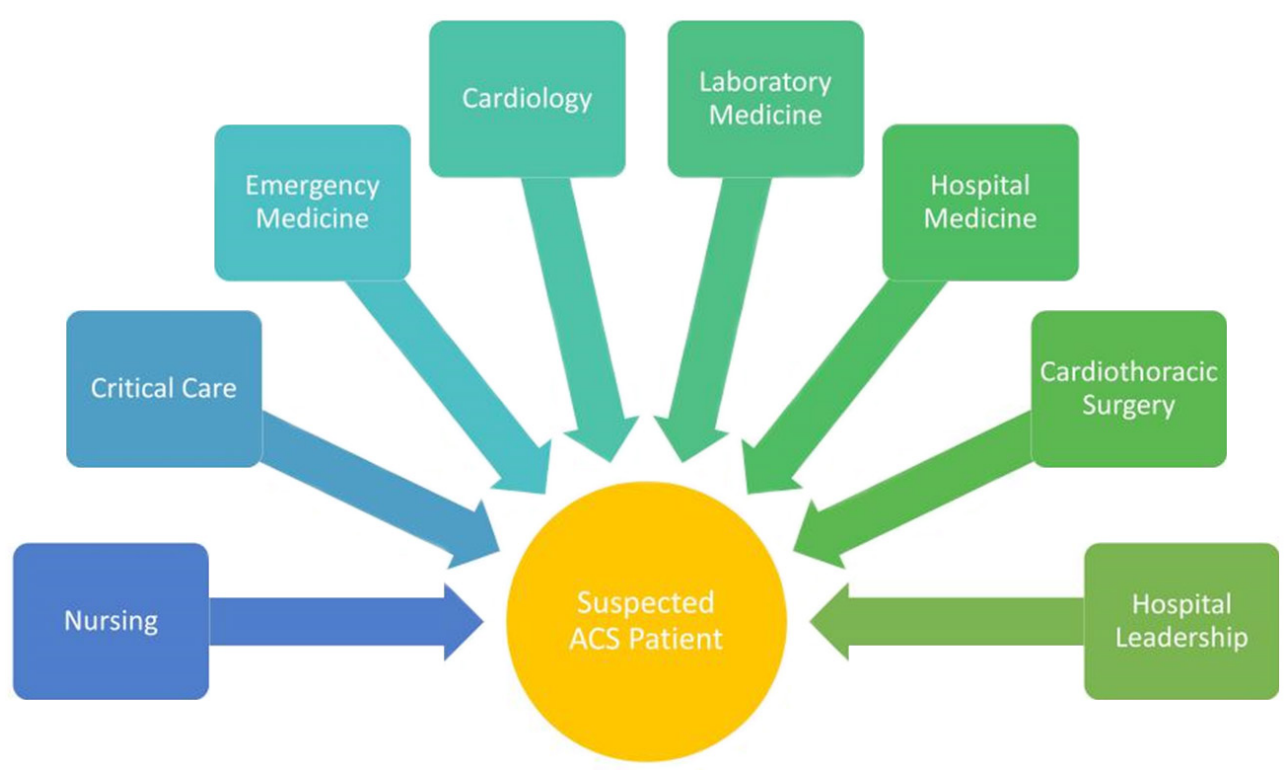

Figure 1. Multidisciplinary approach to the care for patients with suspected acute coronary syndromes (ACS)

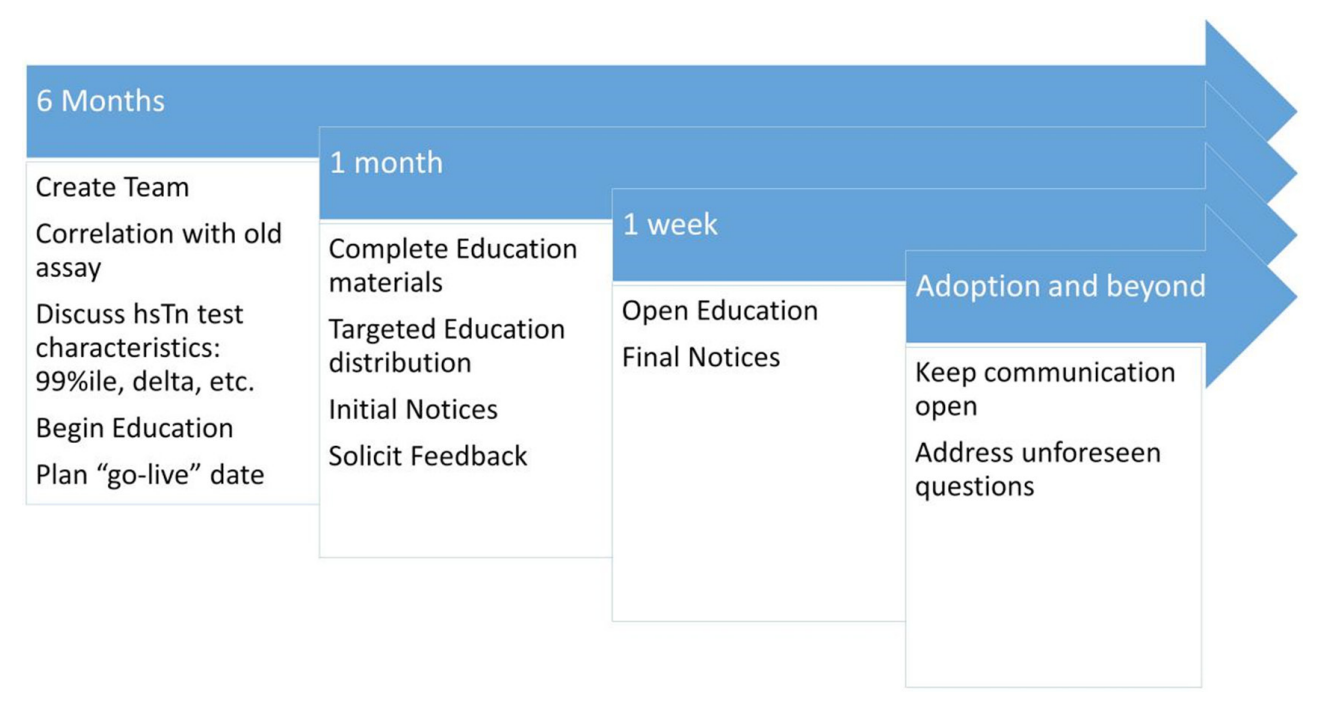

Figure 2. Timeline of activities prior to hsTn adoption

\section{Six months in advance}

At our facility, the change to hsTn was driven primarily by the cardiology and laboratory services. In addition to these groups, the physician champions also recognized the importance of a multidisciplinary approach and made plans to involve a multitude of groups in the implementation [Figure 1]. Our initial planning meetings started approximately 6 months prior to the date we intended to go live with our hsTn assay and a general timeline for the implementation was drafted [Figure 2]. Important tasks included establishing goals for implementation, correlation between the standard and hsTn assays, discussion of cutpoints, and deciding on a "go-live" date. The planning team specifically wanted to focus on implementation on four goals: maximizing sensitivity of the assay, minimizing confusion for clinicians, make adoption as easy as possible for the end users, and to encourage clinicians to use uniform terminology while referring to troponin results. 
Table 1. Recommendations provided for interpretation of hsTn

Serial measurements of high sensitivity troponin (hsTn) are necessary for adequate interpretation and to make clinical decisions; the recommended time interval for serial assays is 0,1 , and $3 \mathrm{~h}$

hsTn result

Interpretation

LESS than or equal to $14 \mathrm{ng} / \mathrm{L}$ (female)

LESS than or equal to $22 \mathrm{ng} / \mathrm{L}$ (male)

Acute cardiac process is highly unlikely (99\% negative predictive

AND NO significant change on serial measurements (increase

or decrease of more than $5 \mathrm{ng} / \mathrm{L}$ )

GREATER than $14 \mathrm{ng} / \mathrm{L}$ (female)

GREATER than $22 \mathrm{ng} / \mathrm{L}$ (male)

AND NO significant change on serial measurements (increase

or decrease of more than $5 \mathrm{ng} / \mathrm{L}$ )

GREATER than $14 \mathrm{ng} / \mathrm{L}$ (female)

GREATER than $22 \mathrm{ng} / \mathrm{L}$ (male)

AND significant change on serial measurements (increase or

decrease of more than $5 \mathrm{ng} / \mathrm{L}$ ) value)

Consistent with chronic (not acute) myocardial damage; this can
be seen in congestive heart failure (CHF), hypertension, chronic
coronary artery disease (CAD), chronic kidney disease (CKD), chronic
obstructive pulmonary disease (COPD), and other conditions
Consistent with acute myocardial damage; this can be seen in acute
coronary syndromes as well as other acute conditions such as
hypertensive emergency, acute exacerbation of CHF, acute pulmonary
embolus, systemic infections such as pneumonia

Laboratory staff took samples from 38 patients who were evaluated with the standard troponin and analyzed them using the hsTn assay [Supplementary Table 1]. Clinical data for the patients were reviewed by cardiology physicians for agreement between the assays. The manufacturer recommended $99^{\text {th }}$ percentile (99\%ile) [> 14 nanograms per liter (ng/L) for women; > $22 \mathrm{ng} / \mathrm{L}$ for men] was used for this exercise and no evidence was observed to justify considering an alternative. While the MRVAMC, like many Veteran-oriented care facilities, care for significantly more men than women, we elected to adopt gender specific 99\%ile thresholds recommended by the manufacturer. Our team, in consultation with hospital medicine, agreed to avoid adoption of the hsTn assay during winter months when volume of influenza cases was anticipated to be highest. We also agreed that when the new assay went live, we would not simultaneously report the standard and hsTn assays to minimize potential confusion if one assay was abnormal and the other was note. Initially, the "go-live" date was scheduled for April 15, 2020; due to the coronavirus pandemic, this was ultimately delayed to June 15, 2020.

Our cardiology service had a detailed discussion regarding the degree of change in hsTn (or "delta") to be considered significant, that would represent a potentially acute cardiac process. Two primary options were considered: using a whole number as recommended by the manufacturer or using a percentage change as recommended in the Universal Definition for Myocardial Infarction ${ }^{[3]}$. At present, all VA health care facilities use the Computerized Patient Record System (CPRS) for electronic medical recordkeeping. Within the laboratory reporting section of the software, there is no functionality to automatically calculate and report changes on serial assays. As such, using or encouraging a percentage change in hsTn would require end users to manually calculate if the values changed by a certain threshold between samples $(20 \%$ change if initial value is below the $99 \%$ ile, $50 \%$ change if the initial value is above the $99 \%$ ile $)^{[3]}$. We acknowledged the difficulty this would pose for individual physicians and elected to report in CPRS that a change of $5 \mathrm{ng} / \mathrm{L}$ may indicate a significant change that would warrant further action. Under each patient's laboratory values, CPRS is capable of displaying a text box with further guidance. The cardiology team drafted text to help explain the importance of serial testing and how to interpret serial values into 1 of 3 categories, all values under the 99\%ile (no cardiac process), values that are above the 99\%ile but not dynamic (chronic myocardial injury), or values above the $99 \%$ ile with dynamic change (acute injury/infarction) [Table 1]. The decision to recommend serial testing at 0,1 , and $3 \mathrm{~h}$ was the consensus of our clinicians based on professional society documents and published literature on similar protocols ${ }^{[2,3]}$. Our team did not elect to use an absolute cutoff to indicate potential ACS. This decision was made, in part, on the experience at UFCOM where a threshold of $100 \mathrm{ng} / \mathrm{L}$ was identified as a "possible myocardial infarction" and led to a number of panicked consults from physicians. Once all these decisions were made, the cardiology team began working on educational materials to inform the facility staff. 
Reporting in whole numbers $(0.03 \mathrm{ng} / \mathrm{mL} \rightarrow 30 \mathrm{ng} / \mathrm{L})$

New assay is more precise and reliable; detectable in $>50 \%$ of controls with no heart disease

Reference values of a control population have an excellent negative predictive value for acute $\mathrm{MI}$ $99.7 \%$

Serial testing is CRUCIAL to separate chronic from acute elevation

Recommended testing interval: 0, 1, $3 \mathrm{~h}$

If $T n$ is elevated and not changing, is due to chronic disease (CAD, CKD, HTN) NOT acute MI

If $T n$ is elevated AND changing, may be due to plaque rupture OR supply/demand mismatch

DON'T use hsTn indiscriminately

--Most ICU patients have elevated hsTn, only order in the ICU if suspicion for MI is HIGH

--No use for hsTn in the outpatient setting, if you suspect MI, send to the ED

CAD: coronary artery disease; CKD: chronic kidney disease; ED: emergency department; hsTn: high sensitivity troponin; HTN: hypertension; ICU: intensive care unit; L: liter; MI: myocardial infarction; mL: milliliter; ng: nanogram

\section{One month in advance}

At this stage, the implementation team reached out to clinical services most likely to be affected by the new hsTn assay: emergency medicine, hospital medicine, critical care and the internal medicine residency. Staff communicated, primarily through email, to identify clinical pathways that would be affected and how to plan for the hsTn change. This broader coalition also identified hospital units and clinical groups that should receive targeted, in-person training on the new assay. E-mail communication and staff meetings were used at this stage to inform front-line clinicians about the hsTn change. Notice of the change was sent to the local members of Congress, in anticipation of any possible concerns from citizens.

Educational materials were finalized with at least a month to go before implementation. The materials started with background on the assay and how sensitivity was improved over the standard assay. This required a brief explanation of concepts such as the limit of detection, limit of quantification, and the 99\%ile. Next discussed was the change in units that would result in whole number reporting. Standard terminology of type 1 myocardial infarction, type 2 myocardial infarction, acute myocardial injury, and chronic myocardial injury were reviewed. Learners were specifically encouraged to avoid non-standard terms such as "troponinemia" and "troponin leak". Data on prevalence, sensitivity, specificity, and positive/ negative predictive values were discussed with a request that clinicians DO NOT order hsTn in the outpatient setting or for inpatients without a high pretest suspicion of an ACS.

Multiple slide presentations were created including a detailed review (approximately $30 \mathrm{~min}$ ), a brief summary (10 min), and a summary specifically for the nursing staff. A single page summary was also created for staff to print and make available in their work areas. Each were uploaded to a shared intranet website so staff could review independently. Versions were created with and without recorded audio of the content. A specialized doctor conducted training sessions with hospital medicine, cardiology, the emergency department, the medical and surgical intensive care units, and one of the general inpatient units where most patients with cardiac diagnoses are grouped geographically.

\section{One week in advance}

The general medical staff (physicians, nurses, and technologists) were informed of hsTn via email from the Chief of Staff's office and in the facility's weekly all-staff newsletter. This notice included an 8-point summary of what the new hsTn assay would mean, the link to more information on the intranet, and an invitation to attend 1 of 4 open educational sessions, hosted by a specialized doctor [Table 2].

\section{RESULTS}

The hsTn assay went live on a Monday morning at $8 \mathrm{AM}$. For the first week, the cardiology service identified additional faculty that would be assigned specifically to address any excess consultations related 


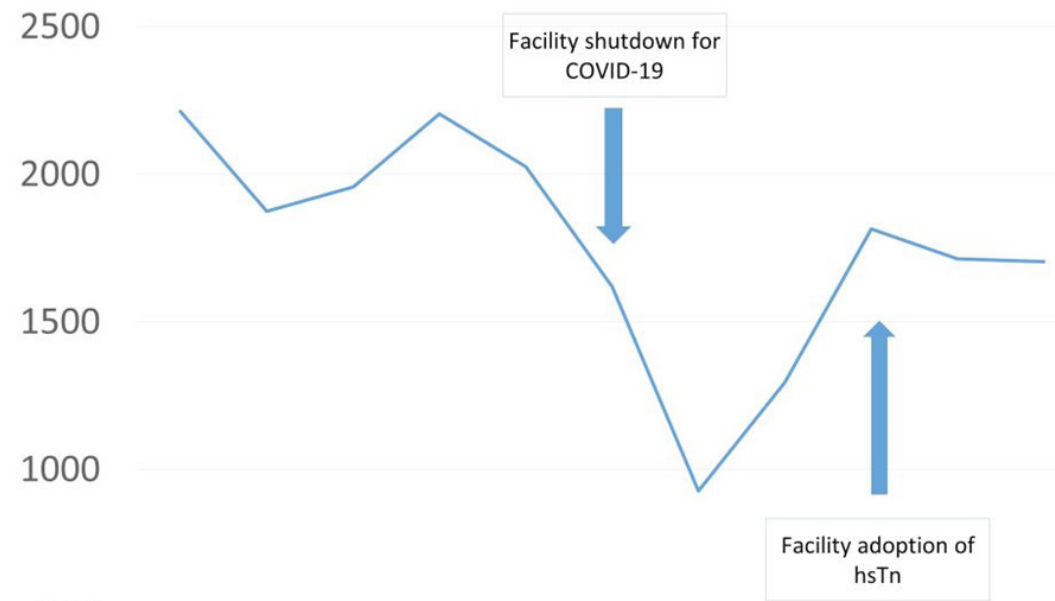

500

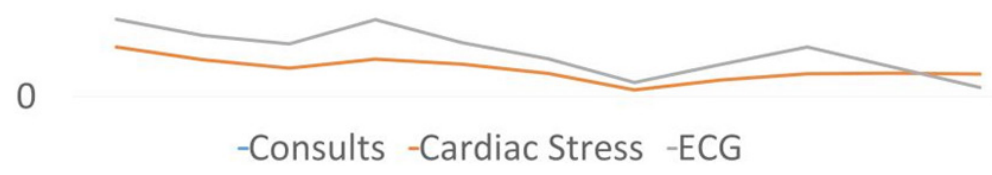

Figure 3. Volume of cardiovascular services before and after hsTn adoption

to hsTn adoption. In the week that followed, the cardiology service received only 2 consultations that were strictly because the ordering physicians were unaware, unfamiliar, or uncomfortable with the hsTn assay. In both cases, the patient had chronic myocardial injury (hsTn $>99 \%$ ile with no change of $>5 \mathrm{ng} / \mathrm{L}$ ) due to underlying medical conditions. Our cardiology team received a call from the nuclear cardiology laboratory after about a week of the new assay initiation. The technologists were unaware of the change and alarmed that a patient with troponin "in the hundreds" would be sent for myocardial perfusion imaging. This group had not been specifically included in our pre-implementation planning or targeted education. No complaints or concerns about the hsTn assay were communicated to the implementation team. Quantitative data on cardiology consultations, electrocardiograms, and stress testing show no changes in demand after adoption of hsTn [Figure 3].

\section{DISCUSSION}

We successfully implemented hsTn in a health system spanning two medical centers and multiple outpatient clinics. By using a multidisciplinary approach, planning well in advance, and using a wide array of educational and communication techniques, the implementation went smoothly. We offer our experience as an example to other institutions that will soon need to adopt a hsTn assay of their own. In a broader sense, the methods used to adopt hsTn at our facility would also be potentially applicable to bringing any new biomarkers on board.

At one time, the medical community had no biomarkers specific to the heart, or even to the muscle with which is used to diagnose critical illnesses like ACS. The first blood test used for making the diagnosis of myocardial injury was aspartate amino transferase, or AST (referred to then as SGOT) ${ }^{[4]}$. In the 1960's and 1970's, creatine kinase isoenzymes and lactate dehydrogenase gained favor. Around this time, the World Health organization published a brief 2 page paper on how to diagnose myocardial infarction (MI), predecessor to the now oft quoted "Universal Definition of MI" ${ }^{[5]}$. It was not until the 1990's when troponin came into use. At each of these stages, clinicians experienced challenges with how to best incorporate new tools into their practice. Inevitably, questions would arise about the utility of the new test and how to best incorporate it into practice ${ }^{[6-8]}$. 
The advent of hsTn mirrors these progressive changes through medical history, but some important differences are worth noting. For one, the infrastructure to conduct large scale studies of new medical advancements has substantially grown. Numerous studies have been published using hsTn to rapidly "rule out" acute $\mathrm{MI}^{[9-12]}$. Another change is the foundation and development of a new discipline called implementation science. This field of study seeks to apply research evidence into practice and has potential application in pragmatic clinical trials, quality improvement, and how to best approach adoption of new clinical practices, such as hsTn.

To achieve best results in spread of knowledge and reproducibility of results, we would have preferred to use formal implementation science methods in adopting hsTn; however, this was beyond local expertise and resource availability. Even without a formal scientific framework, we believe some important lessons can be taken from our experience. Firstly, we strongly encourage facilities to plan months in advance of their hsTn adoption to consider all the important local factors. Secondly, change champions should engage with a wide network of all professionals whose work might be impacted by the new assay including those involved in noninvasive cardiac imaging. Thirdly, education should be provided at multiple levels of detail, made available in-person and on demand, and spread by a variety of communication methods. If available, facilities with communication offices or staff responsible for workforce training should commit time and energy to a smooth transition. Fourthly, staff should be given ample opportunity to be informed about the changes and provide feedback to the champions before, during, and after the process.

In conclusion, with a well-planned multidisciplinary approach, uneventful implementation of hsTn can be achieved in large medical centers.

\section{DECLARATIONS}

\section{Authors' contributions}

The author contributed solely to the article.

\section{Availability of data and materials}

Data can be made available through contact with the author.

\section{Financial support and sponsorship}

Dr. Winchester is supported by Career Development Award (\#13-023) from the United States Department of Veterans Affairs Health Services Research and Development Service.

\section{Conflicts of interest}

The author declared that there are no conflicts of interest.

\section{Ethical approval and consent to participate}

Not applicable. In accordance with VA Handbook 1058.05, this project was performed with the purpose of improving the quality of care and was determined to not qualify as human subjects research.

\section{Consent for publication}

Not applicable.

\section{Copyright}

(c) The Author(s) 2021.

\section{REFERENCES}

1. Anand A, Shah ASV, Beshiri A, Jaffe AS, Mills NL. Global Adoption of High-Sensitivity Cardiac Troponins and the Universal Definition 
of Myocardial Infarction. Clin Chem 2019;65:484-89.

2. Januzzi JL Jr, Mahler SA, Christenson RH, et al. Recommendations for Institutions Transitioning to High-Sensitivity Troponin Testing: JACC Scientific Expert Panel. J Am Coll Cardiol 2019;73:1059-77.

3. Thygesen K, Alpert JS, Jaffe AS, et al; ESC Scientific Document Group. Fourth universal definition of myocardial infarction (2018). Eur Heart J 20182019;40:237-69.

4. Ladenson JH. A personal history of markers of myocyte injury [myocardial infarction]. Clin Chim Acta 2007;381:3-8.

5. Nomenclature and criteria for diagnosis of ischemic heart disease. Report of the Joint International Society and Federation of Cardiology/ World Health Organization task force on standardization of clinical nomenclature. Circulation 1979;59:607-9.

6. Thygesen K. Do new biochemical markers of myocardial damage change the concept of unstable angina pectoris? Eur Heart $J$ 1998;19:1749-50.

7. McQueen MJ, Holder D, El-Maraghi N. Assessment of the accuracy of serial electrocardiograms in the diagnosis of myocardial infarction. Am Heart J 1983;105:258-61.

8. Stuart J. Diagnostic enzymology in myocardial infarction. Am Heart J 1965;70:717-9.

9. Body R, Carley S, McDowell G, et al. Rapid exclusion of acute myocardial infarction in patients with undetectable troponin using a highsensitivity assay. J Am Coll Cardiol 2011;58:1332-9.

10. Haaf $\mathrm{P}$, Reichlin $\mathrm{T}$, Twerenbold R, et al. Risk stratification in patients with acute chest pain using three high-sensitivity cardiac troponin assays. Eur Heart $J$ 2014;35:365-75.

11. Cullen L, Mueller C, Parsonage WA, et al. Validation of high-sensitivity troponin I in a 2-hour diagnostic strategy to assess 30-day outcomes in emergency department patients with possible acute coronary syndrome. J Am Coll Cardiol 2013;62:1242-9.

12. Than M, Aldous S, Lord SJ, et al. A 2-hour diagnostic protocol for possible cardiac chest pain in the emergency department: a randomized clinical trial. JAMA Intern Med 2014;174:51-8. 